\title{
Articulación entre la educación media técnica profesional (EMTP) y la educación superior técnico profesional (ESTP): un análisis de regresiones logísticas estereotípicas
}

\author{
Transition between technical/vocational high school (EMTP) and technical higher \\ education (ESTP): an analysis of stereotypical logistic regressions
}

Claudia P. Ovalle Ramírez' ${ }^{1}$ ORCID: 0000-0002-3664-7290

Recibido: 1/9/2019 • Aprobado: 2/12/2019

Cómo citar: Ovalle Ramírez, C. P. (2020). Articulación entre la educación media técnica profesional (EMTP) y la educación superior técnico profesional (ESTP): un análisis de regresiones logísticas estereotípicas. Ciencia y Educación, 3(3), 21-29. Doi: https://doi. org/10.22206/cyed.2019.v3i3.pp21-29

\section{Resumen}

El presente estudio tiene por objetivo determinar si la educación media técnica - EMTP - tiene un impacto en la probabilidad de selección de programas de educación técnica superior que requieren diferentes niveles de habilidades duras (razonamiento abstracto, razonamiento cuantitativo, pensamiento inferencial y crítico) y blandas (relaciones interpersonales, lenguaje y comunicación, creatividad). Se emplearon regresiones estereotípicas para predecir las probabilidades de matricularse en una escuela de educación superior en comparación con otra, para nueve escuelas (Ingeniería, Turismo, Salud, Construcción, Informática y Telecomunicaciones, Recursos Naturales, Administración, Diseño, Comunicaciones) cuando el estudiante proviene de la media técnica. Los resultados del modelo completo indican que los estudiantes de EMTP tienen mayor probabilidad de elegir programas que exigen un mayor nivel de competencias duras como las Ingenierías de nivel técnico superior.

Palabras clave: enseñanza técnica; enseñanza superior; articulación educativa; elección vocacional.

\begin{abstract}
The present study aims to determine whether technical media education - EMTP - has an impact on the likelihood of selecting higher technical education programs that require different levels of hard skills (abstract reasoning, quantitative reasoning, inferential and critical thinking) and soft skills (interpersonal relationships, language and communication, creativity). Stereotypical regressions were used to predict the odds of enrolling in technical school at a community college compared to another school. The probability of choosing 1 of 9 schools (Engineering, Tourism, Health, Construction, Information Technology and Telecommunications, Administration, Design, Communications) was calculated. The results of the full model indicate that EMTP students may be more likely to choose programs that require a higher level of hard skills (engineering) than soft skills.
\end{abstract}

Keywords: Technical education; higher education; educational articulation; career choice.

a Centro de Justicia Educacional, Pontificia Universidad Católica de Chile. Correo-e: claudia.ovalle@uc.cl 


\section{Introducción}

Chile tiene amplia oferta de educación media y superior técnico-profesional para responder a la necesidad de incrementar la competitividad — por medio de la innovación - y frente a la creciente demanda de acceso a la educación como un derecho social y una aspiración importante de los estudiantes chilenos.

La Educación Técnico Profesional (ETP) en Chile se ofrece en el nivel medio (secundario) y en el superior (terciario) y en ambas instancias, hay oferta pública y privada $^{1}$ (Orellana, 2011; Rucci, Arias, Farías, González-Velosa y Huneeus, 2015). Los oferentes de EMTP -Educación Media Técnico Profesional- son establecimientos de nivel medio y los de la ESTP —Educación Superior Técnico Profesional—son los Centros de Formación Técnica (CFT), Institutos Profesionales (IP) y algunas universidades. Los CFT, IP y universidades confieren títulos de Técnico Superior y los IP y universidades otorgan títulos profesionales. Solo las universidades confieren el grado de licenciado.

Cerca de tres de cada cinco estudiantes de los primeros tres niveles socioeconómicos siguen la modalidad técnico profesional en la enseñanza media (Larragaña, Cabezas y Dussaillant, 2013) concentrando entre el $40 \%$ a $45 \%$ de la matrícula total de los estudiantes de III y IV medio (Agencia de la Calidad, 2016; Sepúlveda y Valdebenito, 2014). Por otra parte, la oferta de ESTP concentra la mayor parte de estudiantes en los CFT y es heterogénea (en número y calidad de programas y titulaciones). Esta oferta no está distribuida uniformemente en las regiones (Larragaña et al., 2013).

En Chile la EMTP se cursa en dos años, después de haber terminado la Enseñanza General Básica (ocho años) y la Enseñanza Media (dos años) que puede ser Científico Humanista (EM-CH) o Técnico Profesional (EM-TP). La formación técnica se aborda desde la perspectiva modular basada en el enfoque de competencias (formación teórica y práctica). La EMTP contempla 44 horas semanales de clases, 24 a 30 del Plan Diferenciado, y el resto del Plan General compartido con la EM-CH (Velasco-Barraza, 2008). Ofrece al final la licencia de enseñanza media, y entrega una certificación de Técnico de Nivel Medio si se cumple con la práctica ${ }^{2}$ en alguna de las 35 especialidades $^{3}$ (Antivilo-Bruna, Poblete-Orellana, Hernández-Muñoz, García y Contreras, 2017). Las ramas que tienen mayor demanda entre los estudiantes son la de Comercial e Industrial y las de menor Agrícola y Marítima (Mineduc, 2011).

Mineduc (Ministerio de Educación de Chile) ha definido el currículo EM-TP por objetivos terminales asociados con el perfil de egreso para cada especialidad. Este currículo fue formulado para lograr concordancia con las necesidades del mercado laboral y las competencias que este reclama (Carrillo y Jurado, 2017). Sin embargo, persiste el debate sobre la relación y continuidad entre la formación media técnica profesional y la formación superior técnica (Sepúlveda, 2013; Sevilla, 2014). En Chile se requiere evidencia actualizada, válida y pertinente que soporte la articulación entre los niveles de EMTP y ESTP. Por ejemplo, es necesario conocer si existe un impacto de la media técnica —EMTP — en las elecciones de formación superior de los estudiantes chilenos.

En Chile hay 949 establecimientos que imparten EM-TP (con un total de 296.596 estudiantes matriculados) (Mineduc, 2011; Riquelme-Brevis, Rivas-Burgos y Riquelme-Brevis, 2018). En la ESTP hay una menor proporción de egresados de la EM-TP (41 \%) y solo un $20 \%$ ingresa al ańo siguiente de haber egresado de la educación secundaria, mientras que en el grupo de la EMCH esta cifra alcanza a alrededor de un 50 \% (Catalán, 2016; Larrañaga et al., 2013). De los egresados EM-TP en 2012 cerca del $80 \%$ continuaron en carreras ESTP. De este total $46 \%{ }^{4}$ ingresó a aquellas carreras directamente relacionadas

\footnotetext{
${ }^{1}$ La educación técnico profesional superior ha sido principalmente de corte privado (Orellana, 2011).

2 Una práctica de 480-960 horas, que por lo general se lleva a cabo al terminar la educación media. En algunos casos, y debido a estos requisitos, los estudiantes no hacen la práctica profesional y no obtienen la licencia de técnico medio (OECD, 2009).

3 Las especialidades ofertadas están agrupadas en las siguientes cinco ramas: Comercial, Industrial, Servicios y Técnica, Agrícola y Marítima.

${ }^{4}$ Aproximadamente esta misma proporción de estudiantes EMTP y ESTP fue hallada en la muestra estudiada por Sepúlveda y Ugalde (2010) quienes reportan un $43,4 \%$.
} 
con sus estudios técnicos de media (Sevilla, Farías y Weintraub, 2014).

El presente estudio tiene por objetivo determinar si la educación EMTP tiene un impacto en la probabilidad de selección de programas de educación técnica superior que requieren diferentes niveles de habilidades duras (razonamiento abstracto, razonamiento cuantitativo, pensamiento inferencial y crítico) y blandas (relaciones interpersonales, lenguaje y comunicación, creatividad).

\section{Muestra}

Se empleó un panel de datos de los años 2015 a 2018 de estudiantes del CFT (Centro de Formación Profesional) e IP (Instituto Profesional) Duoc UC. La muestra comprende 32.676 estudiantes de jornada diurna, de las nueve escuelas de formación técnica superior (Ingeniería, Informática y Telecomunicaciones, Construcción, Recursos Naturales, Salud, Administración, Diseño, Comunicaciones, Turismo). Para estos estudiantes se encuentran datos completos en las variables de nombre de colegio, dependencia del liceo, puntaje NEM —notas de la educación media-, Puntaje Global de PSU, Especialidad de la educación media - técnico profesional o científico humanista-, género, edad, y como identificador individual el RUT. También se conoce la escuela de formación ESTP a la cual se matriculó el estudiante en primer semestre.

\section{Análisis de datos}

Para el análisis de datos se sigue la metodología descrita por Liu (2014) de regresiones estereotípicas, que permite predecir las probabilidades de que un estudiante opte por un programa de formación ESTP en comparación con otro, considerando variables de la educación media como NEM y puntaje PSU y variables de las instituciones escolares como la dependencia del colegio (privado, particular subvencionado y municipal) y el tipo de educación media — técnico profesional o humanista-.

La elección del estudiante por una escuela de formación en la ESTP es la variable dependiente.
Esta variable se considera como una variable ordinal ya que la decisión se centra en una de las escuelas que reúnen los programas técnico profesionales Duoc UC organizadas en un ranking de 0 a 8 dependiendo de su nivel de exigencia de habilidades blandas (Escuela de Turismo, por ejemplo), hasta aquellas que requieren un mayor nivel de competencias duras (Escuela de Ingenierías y Escuela de Informática y Telecomunicaciones). La ordenación de las escuelas en progresión descendente fue la siguiente: Ingeniería (NOMESCU=9), Informática y Telecomunicaciones (NOMESCU=8), Construcción (NOMESCU=7), Recursos Naturales (NOMESCU=6), Salud (NOMES$\mathrm{CU}=5)$, Administración (NOMESCU=4), Diseño (NOMESCU=3), Comunicaciones (NOMESCU=2), Turismo (NOMESCU=1).

La distribución de frecuencias por cada una de estas categorías en el presente estudio es la siguiente:

Tabla 1. Frecuencia y porcentaje de estudiantes de la muestra en cada una de las escuelas

\begin{tabular}{lcc}
\hline $\begin{array}{c}\text { Escuela } \\
\text { (Variable NOMESCU) }\end{array}$ & $\begin{array}{c}\text { Total Estudiantes de la } \\
\text { Muestra }\end{array}$ & $\begin{array}{c}\text { Porcentaje } \\
\%\end{array}$ \\
\hline Ingeniería (9) & 3358 & 10.28 \\
Informática y Telecomunica- & 3021 & 9.25 \\
ciones (8) & & \\
Construcción (7) & 2793 & 8.55 \\
Recursos Naturales (6) & 6586 & 20.16 \\
Salud (5) & 4039 & 12.36 \\
Administración (4) & 1777 & 5.44 \\
Diseño (3) & 3968 & 12.14 \\
Comunicaciones (2) & 3531 & 10.81 \\
Turismo (1) & 3603 & 11.03 \\
\hline
\end{tabular}

Fuente: elaboración propia.

Inicialmente se hizo un modelo de regresión logística en el cual se asume el supuesto de que las probabilidades entre categorías de la variable dependiente son proporcionales (PO o "proportional odds model”), es decir, se asume que los modelos binarios subyacentes, que dicotomizan la variable de respuesta (ordinal), tienen los mismos coeficientes o que los coeficientes logit por cada predictor son los mismos en todas las categorías ordinales. 
El modelo PO, es un modelo de regresión logística que está dado por la ecuación:

$$
\begin{array}{r}
\ln \left(Y_{1}^{\prime}\right)=\operatorname{logit}[\pi(x)]=\ln \left[\frac{\pi_{j(x)}}{1-\pi_{j(x)}}\right]= \\
\alpha+\left(\beta_{1} X_{1}-\beta_{2} X_{2}-\cdots .-\beta_{p} X_{p}\right)
\end{array}
$$

Donde $\pi_{\mathrm{j}(\mathrm{x})}$ es la probabilidad de estar en la categoría J o por debajo de ella dados los predictores X1 a Xp. $\alpha$ representa unos puntos de corte de la variable dependiente y $\beta_{1} \ldots \beta_{p}$ son coeficientes de regresión logística (logit). Se asume que para un predictor solo hay un mismo coeficiente para todas las comparaciones.

Usualmente, el criterio de PO no se cumple. Los modelos logísticos multinomiales de regresión calculan J-1 comparaciones entre las diferentes categorías no ordinales de la variable dependiente permitiendo a su vez que se calculen coeficientes logit por cada predictor. Asimismo, los modelos logísticos estereotípicos se emplean cuando la variable dependiente tiene carácter ordinal, pero se viola el presupuesto de PO. El modelo de regresión logística estereotípica (SL) añade a los modelos multinomiales unas restricciones $(\varphi)$ para asegurar que la variable de respuesta es categórica si se satisface la condición de que:

$$
1=\varphi_{1}>\varphi_{2}>\varphi_{3>\ldots .}>\varphi_{\mathrm{J}}=0
$$

Donde la primera restricción se fija como 1 y la última como 0 para que el modelo SL sea estimado. Si un par de estas restricciones son la misma entonces las categorías se pueden fusionar para hacer los contrastes y para establecer la ordinalidad de la variable. Esto hace que los modelos SL en comparación con los modelos logísticos multinomiales sean más parsimoniosos.

En los modelos SL para calcular las probabilidades de pertenecer a una categoría solo se necesita exponenciar o elevar la base "e" a los coeficientes Beta de la regresión logística, para obtener las probabilidades de pertenecer a una categoría, versus estar en la categoría de comparación o categoría "J" (usualmente la más alta en el orden de la variable dependiente). El modelo de regresión logístico SL está dado por la siguiente ecuación:

$$
\begin{aligned}
\operatorname{logit}[\pi(j, J)] & =\ln \left[\frac{\pi\left(Y=j \perp X_{1}, X_{\left.2, X_{3} \ldots . . . X_{P}\right)}\right.}{\left(Y=J \perp X_{1}, X_{2, X_{3} \ldots . . .} X_{P}\right.}\right] \\
& =\alpha-\varphi_{1}\left(\beta_{1} X_{1}-\beta_{2} X_{2}-\cdots .-\beta_{p} X_{p}\right)
\end{aligned}
$$

Donde, $j=1,2,3 \ldots, J-1, J$, siendo la categoría base de la comparación, por lo general, la categoría más alta de la variable dependiente o "J". Y es la variable de respuesta, $\alpha$ son los interceptos y $\beta$ los coeficientes logit para cada uno de los predictores representados por X. $\varphi \mathrm{j}$ representa las restricciones que hacen de la variable respuesta una variable de tipo ordinal.

Para el análisis de este estudio se usó el comando ologit de Stata 14.0 para ajustar un modelo de regresión logística PO bajo el supuesto de que los coeficientes no varían en los niveles de la variable categórica. A continuación, se probó el supuesto de PO, por medio del test de Brant. Luego se desarrollaron dos modelos de regresión estereotípica (LS). El primero incluye solo una variable y el segundo incluye todas las variables (Paquete Spost de Stata). Los outputs del software Stata se incluyen en los anexos 1 a 3 .

\section{Resultados}

\section{Modelo PO con variables explicativas}

El primer modelo del anexo 1 se empleó como modelo logístico de regresión de las variables tipo de liceo (privado, municipal/público, privado subsidiado), NEM (promedio de las notas de media), Puntaje Global PSU (prueba de admisión a la universidad) y tipo de enseñanza media (técnico o científico humanista) sobre la elección del estudiante por una de las nueve escuelas de formación técnica. El test $\chi^{2}$ de para la probabilidad log (log likelihood) equivale a -69300.109, $\mathrm{p}=0.000$. Indicando que el modelo de 4 predictores es mejor que el modelo nulo sin ninguno.

Los efectos logit para cada uno de los predictores sobre la variable respuesta fueron significativos a excepción del tipo de colegio municipal y el promedio PSU. El coeficiente logit de regresión estimado para tipo de enseñanza media técnico profesional fue $\beta=0.304, z=14.29, p<0.001$; para tipo de colegio particular $\beta=-0.706, \mathrm{z}=-19.66, \mathrm{p}<0.001$; para tipo 
de colegio municipal $\beta=0.015, \mathrm{z}=-0.65, \mathrm{p}<0.517$; para NEM $\beta=-0.284, z=-10.35, p<0.001$ y para PSU Global $\beta=-0.00003, z=-0.26, p=0.796$. Esta evidencia indica que la educación media tiene efectos en las probabilidades de que los estudiantes elijan carreras técnicas y profesionales que demandan habilidades progresivamente más "duras". En términos de razón de las probabilidades (odds ratio, OR), las posibilidades de que un estudiante esté matriculado en una escuela de mayor exigencia de habilidades duras es 1.35 veces más grande cuando proviene de EMTP y 1.05 veces más grande cuando proviene de un colegio municipal, comparado con un colegio privado (0.49). Sin embargo, cuando los estudiantes tienen un NEM más alto y un promedio PSU también más alto tienen mayor probabilidad de matricularse en escuelas de ESTP que no les demandan habilidades duras (ORS=0.752 y 0.999, respectivamente).

\section{Test de Brent del supuesto de proporcionalidad de las probabilidades}

El modelo anterior debe revisarse para establecer si cumple con el supuesto de proporcionalidad de probabilidades. El test de Brant que se presenta en la tabla 2 indica que se ha violado el supuesto ya que es significativo estadísticamente. Por esto se justifica el uso de un modelo de regresión logística SL que puede hacer variar las $\mathrm{OR}$ encontradas en el modelo ordenado logístico del Anexo 1.

Tabla 2. Test del supuesto de regresiones paralelas

\begin{tabular}{r|ccc} 
& Chi2 & df & P>Chi2 \\
\hline Wolfe Gould & 1708 & 35 & 0.000 \\
Brant & 1747 & 35 & 0.000 \\
score & 1727 & 35 & 0.000 \\
Wikelihood ratio & 1578 & 35 & 0.000 \\
Wald & 2526 & 35 & 0.000
\end{tabular}

Fuente: elaboración propia.

\section{Modelo de Regresión Logística estereotípica (LS) para una variable}

Debido a que la variable de interés es la participación del estudiante en EMTP antes de matricularse en ESTP, se emplea la variable Tipo de Educación Media (técnico profesional o científico humanista) como variable predictor en el modelo LS. Para este modelo el test Chi cuadrado de Wald con un grado de libertad equivale a $\chi^{2}=187.16 \mathrm{p}<0.001$, indicando que el coeficiente logit para el predictor es estadísticamente diferente de 0 . El coeficiente logit estimado $\beta=0.710, z=13.68, p<0.001$ indica que el tipo de educación media (técnico profesional) tiene una relación significativa con el tipo de escuela ESTP en la cual se matricula el estudiante.

En este modelo, las probabilidades de estar en una escuela que demanda habilidades duras (categoría 9 ingenierías) comparado con matricularse en una escuela ESTP que exige habilidades más blandas (categoría 1 o turismo) equivale a OR $(1,9)=\mathrm{e}^{-\Phi \times \beta-1}$, lo que es igual a $\operatorname{OR}(1,9)=\mathrm{e}^{-1 \times 0,710}=0.49^{5}$. El resultado indica que la probabilidad de estudiar una carrera ESTP en una escuela que demanda más habilidades blandas (turismo) comparado con la escuela que demanda más habilidades duras (ingenierías) es 0.49 veces mayor cuando se proviene de la educación EMTP (educación media técnico profesional).

Ya que la segunda restricción es $\Phi 2=1,21$, las posibilidades de estar en la escuela de comunicación comparado con matricularse en la escuela de ingeniería cuando se proviene de la EMTP es OR $(2,9)=$ $\mathrm{e}^{-1.21 \times 0,710}=0,42$ veces mayor. De igual manera ya que $\Phi 3=1,56$; $\Phi 4=0,60 ; \Phi 5=0,30 ; \Phi 6=0,42 ; \Phi 7=$ 0,$31 ; \Phi 8=0,41$, respectivamente, los OR (odds ratios) obtenidos son: $\mathrm{OR}(3,9)=0,33$; OR $(4,9)=0,65$; OR $(5,9)=0,80 ;$ OR $(6,9)=0,74 ; \mathrm{OR}(7,9)=0,80 ;$ OR $(8,9)=0,74$. Estos resultados significan que cuando se proviene de la EMTP en lugar de matricularse en un programa de la escuela de ingenierías (categoría 9) se aumentan las posibilidades de ingresar a otras escuelas: a la escuela de diseño por un factor de 0,33 $(\mathrm{OR}(3,9))$; a la escuela de administración por un factor

\footnotetext{
${ }^{5}$ Esto es exponenciar el valor inverso (negativo) de la restricción para la primera categoría y el coeficiente de la variable predictora.
} 
de $0,65(\mathrm{OR}(4,9))$; a la escuela de Salud por 0,80 $(\mathrm{OR}(5,9))$; a la de recursos naturales por $0,74(\mathrm{OR}$ $(5,9))$; a la escuela de Construcción por 0,80 (OR $(6,9))$ y a la de informática por $0,80(\mathrm{OR}(7,9))$.

Por último, la probabilidad de que un estudiante se matricule en la categoría más alta (categoría 9 o escuela de Ingeniería) por comparación con la categoría más baja — que requiere habilidades más blandases $\operatorname{OR}(9,1)=2,03$. Esto significa que la probabilidad de que el estudiante que proviene de la EMTP se matricule en la escuela de turismo en lugar de la escuela de Ingeniería $(\mathrm{OR}(9,1))$ es 2,03 veces más alta.

Este modelo de regresión logística solo incluye el predictor de tipo de enseñanza media EMTP. A continuación, se presentan los resultados del modelo cuando se introducen más variables de control.

\section{Modelo Completo (Full) de regresión logística estereotípica con 4 predictores}

El modelo completo se muestra en el anexo 3. Se ajustó este modelo con las variables NEM (promedio de notas), puntaje global PSU, especialidad (tipo de educación media: científico humanista o técnico profesional), tipo de colegio (dependencia: privado, privado subvencionado o municipal). Los estadísticos descriptivos para estas variables se presentan en la tabla 3 y 4 .

Tabla 3. Estadísticos descriptivos variables continuas

\section{SD Min. Max.}

$\begin{array}{lcccc}\text { NEM } & 5.448 & 0.384 & 4 & 6.9 \\ & & & & \\ \begin{array}{l}\text { Puntaje } \\ \text { Global PSU }\end{array} & 510.131 & 72.102 & 24 & 862 \\ & & & & \end{array}$

Nota: $\mathrm{n}=32667$ estudiantes.

Fuente: elaboración propia
Tabla 4. Estadísticos descriptivos variables categóricas

\begin{tabular}{lcc}
\hline & Frecuencia & $\%$ \\
\hline $\begin{array}{l}\text { Dependencia de la Escuela/Tipo Colegio } \\
\text { Particular }\end{array}$ & & \\
Particular Subvencionado & 22021 & 9.25 \\
Municipal/Público & 7610 & 67.47 \\
Tipo de Educación Media & & \\
Técnico Profesional & 21704 & 66.42 \\
Científico Humanista & 10973 & 33.5 \\
Sexo & & \\
Femenino & 13818 & 42.29 \\
Masculino & 18859 & 57.71 \\
Estado Civil & & \\
Casado & 146 & 0.45 \\
Soltero & 32478 & 99.55 \\
Año de Ingreso ESTP & & \\
2014 & 7224 & 22.11 \\
2015 & 10777 & 32.98 \\
2016 & 4401 & 13.47 \\
2017 & 1596 & 4.88 \\
2018 & 761 & 2.33 \\
\hline
\end{tabular}

Nota: $\mathrm{n}=32667$ estudiantes

Fuente: elaboración propia.

El modelo presenta un ajuste adecuado según el estadístico Chi cuadrado de Wald, Wald $\chi^{2}=168.31$, $\mathrm{p}<0.001$, indicando que el modelo con todas las variables provee un mejor ajuste que el modelo de una sola variable.

Los coeficientes logit del modelo LS con todas las variables comparan la probabilidad de estar en la categoría "J" o categoría de base (Escuela de Ingeniería o categoría 9) contra estar en otras categorías (otras escuelas que se rankean en las categorías entre 1 y 8 dependiendo su nivel de exigencia de habilidades duras, siendo 9 el nivel más alto).

Los efectos logit para todos los predictores sobre la escuela técnica superior en la cual se matricula el estudiante fueron significativos a excepción de la variable tipo de colegio municipal ( $p=0.889$ ). El coeficiente de la variable tipo de educación media técnico profesional 
es $\beta=0.254, \mathrm{z}=6,89, \mathrm{p}<0.001$, el cual se redujo comparado con el coeficiente del modelo de regresión LS de una sola variable en el que el coeficiente estimado fue $\beta=0.710, z=13,68, p<.001$. Sin embargo, en ambos modelos el coeficiente de la variable educación media técnico-profesional es significativo, lo cual indica que hay una relación entre este tipo de formación y las elecciones de los estudiantes (el tipo de escuela de formación técnica superior en la que se matriculan).

El coeficiente logit para tipo de colegio de dependencia particular es $\beta=-0.664, \mathrm{z}=-11.27, \mathrm{p}<0.001$, mientras que para el tipo de colegio municipal es $\beta=-0.002, z=-0.14, p=0.889$ por lo que la dependencia escolar se relacionaría con elecciones de carreras menos enfocadas en las habilidades duras (Ingenierías) ya que ambos coeficientes son negativos y marginales (pequeños).

Para el promedio de notas de la educación media NEM el coeficiente logit es $\beta=0.149$, $z=5.86$, $\mathrm{p}<0.001$ y para PSU Global $\beta=-0.002, \mathrm{z}=-10.81$, $\mathrm{p}<0.001$. Ambos coeficientes son significativos estadísticamente, pero en este modelo a diferencia del modelo del anexo 1, el NEM tiene una relación positiva con las probabilidades de que el estudiante escoja una carrera técnica que exija mayores habilidades duras (Escuela de Ingenierías).

En términos de proporción de probabilidades o Odds Ratio (OR), las probabilidades de matricularse en la escuela de ingeniería versus otras escuelas que exigen un menor nivel de competencias duras (optar por el nivel 9 "Escuela de Ingeniería" versus los niveles 1 a 8 que se refieren a otras escuelas) se aumentan -manteniendo las demás variables constantes-. Estos aumentos de las probabilidades se dan por un factor de 0.77 cuando la escuela media es técnico profesional; por 1.93 cuando el colegio es particular, por 1.002 cuando el colegio es municipal y por 1.00 cuando hay un aumento de una unidad en la prueba PSU. Cuando el aumento se da en una unidad del NEM (notas promedio de la educación media), la probabilidad de matricularse en la escuela de Ingeniería en la ESTP es de 1.160. Las probabilidades de 1.0 son muy fuertes e indican que el evento es altamente posible de ocurrir. Es decir, que entre más alto el OR mayores son las probabilidades de que este evento se presente a que no se presente.

$\mathrm{Al}$ exponenciar (elevar el número "e" a la potencia de $-\Phi$ multiplicado por $\beta$ ) se obtienen todos los OR de cada una de las variables predictoras. Para cada una de estas variables se obtienen las probabilidades de que el estudiante se encuentre en la categoría de base "J" (Escuela de Ingeniería) versus cualquier otra de las categorías restantes. La tabla 5 presenta a continuación las OR comparando la categoría de base contra las otras categorías para los 4 predictores del modelo LS.

Tabla 5. OR (odds ratio) para las cuatro variables predictoras por comparación entre categorías ( $Y=J$ vs. $Y=j)$

\begin{tabular}{|c|c|c|c|c|c|c|c|c|}
\hline & \multicolumn{8}{|c|}{ Comparaciones } \\
\hline & $\begin{array}{c}Y=1 \\
V s . \\
Y=9\end{array}$ & $\begin{array}{c}Y=2 \\
V s . \\
Y=9\end{array}$ & $\begin{array}{c}Y=3 \\
V s . \\
Y=9\end{array}$ & $\begin{array}{c}Y=4 \\
V s . \\
Y=9\end{array}$ & $\begin{array}{c}Y=5 \\
V s . \\
Y=9\end{array}$ & $\begin{array}{c}Y=6 \\
V s . \\
Y=9\end{array}$ & $\begin{array}{c}Y=7 \\
V s . \\
Y=9\end{array}$ & $\begin{array}{c}Y=8 \\
V s . \\
Y=9\end{array}$ \\
\hline EMTP & 0.775 & 0.681 & 0.739 & 0.924 & 1.175 & 0.975 & 1.105 & 0.848 \\
\hline Particular & 1.942 & 2.729 & 2.269 & 1.228 & 0.655 & 1.067 & 0.769 & 1.536 \\
\hline Municipal & 1.002 & 1.003 & 1.002 & 1.006 & 0.998 & 1.000 & 0.999 & 1.001 \\
\hline NEM & 1.160 & 0.798 & 1.201 & 1.047 & 1.099 & 0.985 & 1.060 & 0.908 \\
\hline PSU & 1.002 & 1.003 & 1.002 & 1.006 & 0.998 & 1.000 & 0.999 & 1.001 \\
\hline
\end{tabular}

Fuente: elaboración propia. 
Nota1: Escuela de Ingeniería $(Y=9)$, Informática y Telecomunicaciones $(Y=8)$, Construcción $(Y=7)$, Recursos Naturales $(Y=6)$, Salud $(Y=5)$, Administración $(Y=4)$, Diseño $(Y=3)$, Comunicaciones $(Y=2)$, Turismo $(Y=1)$. Nota2: La categoría de base $(\mathrm{J})$ es la $Y=9$, que se refiere a la escuela de Ingenierías.

Las probabilidades OR muestran que cuando el estudiante proviene de educación técnico profesional (EMTP) se incrementa la probabilidad de que se matricule en programas técnico profesionales que requieren competencias duras (categoría 9) comparado con la opción de elegir programas que requieren competencias blandas. El mismo patrón de probabilidades se observa con relación a la dependencia del colegio, el puntaje NEM y la prueba PSU.

\section{Conclusión}

La formación media tiene un impacto en el tipo de educación superior técnico profesional elegida por los estudiantes. El análisis presentado indica que los estudiantes de EMTP tienen mayor probabilidad de elegir programas que exigen un mayor nivel de competencias duras como las Ingenierías de nivel técnico superior. Debido a la poca evidencia existente sobre los impactos de la educación media en la formación superior, este resultado debe contrastarse en estudios futuros.

El estudio también muestra evidencia de que la formación superior técnica en programas con mayor exigencia académica está relacionada con el rendimiento en la educación media y con las habilidades del estudiante obtenidas en la educación media y medidas con las pruebas PSU (el equivalente del SAT norteamericano). Asimismo, el tipo de colegio del que proviene el estudiante privado o municipal se relaciona con una elección de programa técnico superior más relacionado con competencias blandas (ejemplo, turismo). Este resultado puede estar explicado porque son pocos los estudiantes de educación privada que eligen la educación técnica y porque se ha identificado que los estudiantes de educación municipal tienden a presentar un nivel de desempeño menor que el de sus pares en educación privada y subvencionada.

\section{Reconocimientos}

\section{Proyecto PIA CIE CONICYT 160007.}

\section{Referencias}

Agencia de la Calidad de la Educación. (2016). Panorama de la educación media técnico profesional en Chile 2016. Santiago de Chile, Chile: Autor.

Antivilo-Bruna, A., Poblete-Orellana, V., Hernández-Muñoz, J., García, C., y Contreras, P. (2017). Factores individuales, sociodemográficos e institucionales en el acceso de los egresados de la educación media técnico profesional a las instituciones de educación superior. Calidad en la Educación, 46, 96-132.

Carrillo, O., y Jurado, P. (2017). La educación técnico profesional y las competencias para la ciudadanía. El caso de las comunas de la provincia de Concepción, Chile. Calidad en la Educación, 46, 133-164.

Catalán, X. (2016). Elección de modalidad educativa en la enseñanza media y su rol en la postulación a las universidades del CRUCH. Calidad en la Educación, 45, 288-320

Larrañaga, O., Cabezas, G., y Dussaillant, F. (2013). Informe completo del Estudio de la Educación Técnico Profesional. PNUD-Área de Reducción de la Pobreza. Recuperado de: http://www.cl.undp. org/content/dam/chile/docs/pobreza/undp_cl_ pobreza_informe_completo_ETP.pdf

Liu, X. (2014). Fitting estereotipe logistic regression models for ordinal response variables in educational research. Journal of modern applied statistical methods, 13(2), 31. Doi: 10.22237/ jmasm/1414816200

Mineduc. (2011). Educación técnico profesional en Chile: Antecedentes y claves de diagnóstico. Santiago de Chile, Chile: Centro de Estudios Mineduc.

Orellana, N. (2011). Educación superior y la pirámide: direcciones de desarrollo del sector no universitario en: Argentina, Chile y Perú. Calidad en la Educación, 34, 48 - 72.

Riquelme-Brevis, H., Rivas-Burgos,M., y Riquelme-Brevis, M. (2018). Criterios de empleabilidad en la educación técnico-profesional. Educare, 22(2), 1-25. 
Rucci, G. Arias, E. Farías, M., González-Velosa, C., y Huneeus, C. (2015). Educación técnico profesional en Chile. Washington, Estados Unidos: BID.

Sepúlveda, L. (2013). ¿Una Reforma para Fortalecer la Educación Técnico Profesional? Santiago de Chile, Chile: Facultad de Educación Universidad Alberto Hurtado.

Sepúlveda, L., y Ugalde, P. (2010). Trayectorias disímiles y proyectos individualizados: origen y experiencia educativo-laboral de los estudiantes de centros de formación técnica. Calidad en la Educación, (33), pp. 63-99. Doi: https://doi. org/10.31619/caledu.n33.139

Sepúlveda, L. y Valdebenito, M.J. (2014). ¿Las cosas claras? Aspiraciones de futuro y proyecto educativo laboral de jóvenes estudiantes secundarios. Estudios Pedagógicos, 40(1), pp.243-261.
Sevilla, M.P. (2014). La educación técnica en Chile y Estados Unidos desde una perspectiva histórica y comparada. Calidad en la Educación, 40, 298-317.

Sevilla, M.P., Farías, M., y Weintraub, M. (2014). Articulación de la educación técnico profesional. Calidad en la Educación, 41, 83-117

Velasco-Barraza, C. (2008). Tres experiencias exitosas de Chile en la educación técnico-profesional de nivel medio. Santiago de Chile, Chile: OREALC/ UNESCO. 